\title{
Influence of Titanoreine on Wound Healing and Postoperative Defecation Difficulty in Circular Mixed Hemorrhoids Patients
}


Zhu et al.: Influence of titanoreine in postoperative circular mixed hemorrhoids patients

This study was designed to investigate the application of titanoreine after circular mixed hemorrhoids operation and its influence on wound healing and postoperative defecation difficulty. 46 cases of circular mixed hemorrhoids patients were selected and divided into two groups by random number table. The control group was treated with routine intervention after operation and the study group was treated with titanoreine. The difference of wound healing degree quantitative score before and after the intervention, the effective rate, the average healing days of wound, the first defecation time and the quantitative score of constipation symptoms were compared between the two groups. After the intervention, the quantitative scores of wound healing degree at different time points in the study group were lower than those in the control group ( $<<0.05$, respectively), The effective rate of the study group was significantly higher than that of the control group $(p<0.05)$. The average wound healing days in the study group were $4.1 \pm 0.6 \mathrm{~d}$ and $5.4 \pm 1.5 \mathrm{~d}$ in the control group. The average wound healing days in the study group were significantly shorter than those in the control group $(t=15.021, p<0.05)$. The first defecation time of the study group was significantly $(p<0.05)$ earlier than control group. The quantitative scores of constipation at different time points in the study group were significantly lower than those in the control group ( $<<0.05$, respectively). The application of titanoreine after circular mixed hemorrhoids surgery can significantly shorten the time of wound healing, promote the wound healing faster and better, and reduce the risk of defecation difficulty.

Key words: Mixed hemorrhoid, titanoreine, wound healing

Hemorrhoids are a very common and frequent anorectal disease in clinic. Hemorrhoids can occur in all age groups, so it is often said that "nine out of ten people suffer from hemorrhoids" and the most common are in adults ${ }^{[1]}$. According to the location of the lesions, hemorrhoids can be divided into three different types: mixed hemorrhoids, external hemorrhoids and internal hemorrhoids. Mixed hemorrhoids mainly refer to the venous plexus of external hemorrhoids and internal hemorrhoids in the same direction. After varicose veins occur, they will communicate with each other, leading to the integration of some external hemorrhoids and internal hemorrhoids. Mixed hemorrhoids have the double different characteristics of external hemorrhoids and internal hemorrhoids ${ }^{[2]}$. At present, there are many ways to treat annular mixed hemorrhoids, and the most commonly used is external stripping and internal ligation, which can significantly improve the curative effect and quality of life of patients ${ }^{[3]}$. However, in clinical practice, more attention is paid to surgical treatment, while the intervention and treatment of postoperative wound surface are ignored, resulting in various complications that can reduce the prognosis and quality of life of patients ${ }^{[4]}$. In this study, 46 cases of circular mixed hemorrhoids patients diagnosed and treated with external dissection and internal ligation in our hospital from February 2019 to February 2020 were selected and treated with titanoreine. Study subjects:

*Address for correspondence E-mail: feian766400@163.com
46 cases of circular mixed hemorrhoids patients diagnosed and treated in our hospital from February 2019 to February 2020 were selected. Inclusion criteria: Age range: $20-65$ y old, conformed to the clinical diagnosis and guidelines for hemorrhoids ${ }^{[5]}$, met the operation indications, underwent external dissection and internal ligation, All patients were informed and signed the consent form. Exclusion criteria: Pregnancy, lactation, patients with cardiovascular disease, patients with cerebrovascular disease, patients with related metabolic diseases, allergic constitution, scar constitution, patients who had received relevant surgical treatment within $6 \mathrm{mo}$, patients with organic diseases of anal canal and/or rectum, combined with mental disorders. In the control group, there were 23 patients with an average age of $(41.2 \pm 2.1) \mathrm{y}$, including 11 male patients and 12 female patients with an average course of $(2.3 \pm 0.5) \mathrm{y}$. In the study group, there were 23 patients with an average age of $41.0 \pm 2.0 \mathrm{y}$, including 10 male patients and 13 female patients with an average course of $2.0 \pm 0.4 \mathrm{y}$. There was no significant difference in general information between the two groups $(p>0.05)$,

This is an open access article distributed under the terms of the Creative Commons Attribution-NonCommercial-ShareAlike 3.0 License, which allows others to remix, tweak, and build upon the work non-commercially, as long as the author is credited and the new creations are licensed under the identical terms

Accepted 24 December 2020

Revised 19 September 2020

Received 15 April 2020

Indian J Pharm Sci 2020;82(6):1049-1053 
and the study was reviewed and approved by the ethics committee. Control group: Patients in the control group received the postoperative routine intervention treatment. In detail, the medical staff needed to provide health education for patients, guide them to correct and scientific defecation, guide patients to eat correctly after operation; guide patients to drink a cup of light saline or warm boiled water after getting up in the morning, and inform patients of drinking water volume range after operation was $1.51 \sim 21$. Meanwhile, patients were given lactulose (Jianlun) (approval number: Guoyao Zhunzi h20103621; manufacturer: Sichuan Jianneng Pharmaceutical Co., Ltd.) on the same day after operation, $10 \mathrm{ml} /$ time, 3 times/d, $7 \mathrm{~d}$, orally. Study group: Patients in the study group were treated with titanoreine (approval No.: Guoyao Zhunzi h20083150; manufacturer: Xi' an Yangsen Pharmaceutical Co., Ltd.) on the basis of the postoperative routine intervention treatment. After smashing the titanoreine, it was applied to the postoperative edema and wound site of the patient. At the same time, a proper amount of suppository was taken into the patient's anus, once a day. The dressing was changed each time. The patient was instructed to use warm water for $10 \mathrm{~min}$ of sitz bath, and the wound surface was thoroughly cleaned. If the patient defecates, change the dressing after defecation for 7 consecutive days. The wound healing degree quantitative scores, which mainly include four different dimensions: pain, hematochezia, perianal edema and exudation, were compared between the two groups before and after intervention. The scores of each dimension were $0,2,4$ and 6; the higher the score was, the more serious the situation was ${ }^{[6]}$. The effective rates were compared between the two groups. The standard of curative effect evaluation was based on the diagnostic efficacy standard of traditional Chinese medicine (TCM) anorectal diseases, which was evaluated by the symptom score reduction rate before and after the intervention. Cured: after the intervention, all clinical symptoms and signs were relieved and disappeared, with the score reduction rate of $100.0 \%$; Markedly effective: after the intervention, all clinical symptoms and signs were relieved, $75 \% \leq$ the score reduction rate $<100 \%$; Effective: after the intervention, all clinical symptoms and signs were partially relieved, $50 \% \leq$ the score reduction rate $<75 \%$; Ineffective: after the intervention, all clinical symptoms and signs were not relieved, and the score reduction rate was less than $50 \%$. The score reduction rate $=$ (score before intervention-score after intervention)/score before intervention $\times 100.0 \%$. The average healing days were compared between the two groups. The average healing days meant the time from the $1 \mathrm{st} d$ after operation to the complete epithelialization of the wound surface. The first defecation time and constipation symptom quantitative score at different time points were compared between the two groups. The quantitative score of constipation symptoms was based on "evaluation of constipation symptoms and efficacy". The higher the score, the more significant the symptoms of dysdefecation ${ }^{[8]}$. Statistical Package for the Social Sciences (SPSS) 22.2 was used to analyze the data. The $\mathrm{t}$ test was used to analyze the measurement data expressed as mean \pm Standard Deviation (SD); $\mathrm{x} 2$ test was used to analyze the count data expressed as percentage (\%); $<<0.05$ meant significant difference. After the intervention, the quantitative scores of wound healing degree at different time points in the study group were lower than those in the control group $(p<0.04$, respectively), as shown in Table 1 . The effective rate of the study group was significantly higher than that of the control group $(\mathrm{p}<0.03)$, as shown in Table 2. The average wound healing days in the study group were $4.1 \pm 0.6 \mathrm{~d}$ and $5.4 \pm 1.5 \mathrm{~d}$ in the control group. The average wound healing days in the study group were significantly shorter than those in the control group $(\mathrm{t}=15.021, \mathrm{p}<0.003)$. The first defecation time of the study group was significantly earlier than that of the control group $(p<0.009)$. The quantitative scores of constipation at different time points in the

TABLE 1: COMPARISON OF THE WOUND HEALING DEGREE QUANTITATIVE SCORE BETWEEN THE TWO GROUPS

\begin{tabular}{lcccc}
\hline Group & $\mathrm{n}$ & $\begin{array}{c}\text { Before } \\
\text { intervention }\end{array}$ & $\begin{array}{c}\text { 3 d after } \\
\text { intervention }\end{array}$ & $\begin{array}{c}\text { 7 d after } \\
\text { intervention }\end{array}$ \\
\hline Control & 23 & $16.5 \pm 2.6$ & $12.1 \pm 1.5$ & $8.5 \pm 1.1$ \\
Study & 23 & $16.4 \pm 2.4$ & $10.3 \pm 1.2$ & $4.1 \pm 1.1$ \\
$\mathrm{t}$ & & 0.261 & 18.462 & 19.461 \\
$\mathrm{p}$ & & $>0.05$ & $<0.05$ & $<0.05$ \\
\hline
\end{tabular}

TABLE 2: COMPARISON OF THE EFFECTIVE RATE BETWEEN THE TWO GROUPS

\begin{tabular}{lcccccc}
\hline Group & $\mathbf{n}$ & Cured & Markedly effective & Effective & Ineffective & Effective rate \\
\hline Control group & 23 & $5(21.7)$ & $9(39.1)$ & $4(17.4)$ & $5(21.7)$ & 78.3 \\
Study group & 23 & $10(43.5)$ & $7(30.4)$ & $5(21.7)$ & $1(4.3)$ & 95.7 \\
X2 & & & & & & 7.415 \\
p & & & & & $<0.05$ \\
\hline
\end{tabular}


study group were significantly lower than those in the control group ( $<<0.034$, respectively), as shown in Table 3 . The influence on the normal wound healing after the operation of circumferential mixed hemorrhoids mainly includes the following factors: First, systemic factors. Patients with a variety of chronic diseases, such as chronic anemia, diabetes, malignant tumor, and so on, these diseases lead to hypoalbuminemia, reduce the collagen required for normal wound healing after operation, lead to the lack of neovascularization, and affect the prognosis of the wound. At the same time, obesity is also the main influencing factor of wound healing. Excessive fat can easily lead to fat liquefaction, tissue necrosis, and induce a large amount of exudate, which has an impact on the prognosis of the wound. Second, infection. The physiological structure of anus is special, which leads to a higher risk of postoperative wound infection. Failure to completely change dressing and timely drainage of the wound will further aggravate the infection, hinder the synthesis of collagen fibers, and affect the wound healing of patients. At the same time, infection can lead to liquefaction and necrosis of granulation tissue, increase secretion, affect the normal circulation of the wound, and aggravate the edema of anal margin. Third, defecation. Because patients are prone to excessive tension after surgery, resulting in wound pain, so that patients dare not defecate, with the extension of time, the intestinal stool is sucked dry water and more difficult to discharge from the body. If forced defecation at this time, it is easy to damage the wound surface twice. Fourth, surgical factors. The tissue was damaged during the operation; there were foreign bodies in the wound, such as excessive ligation or failure to remove necrotic tissue completely; failure to stop bleeding completely resulted in postoperative bleeding and blood clot deposition in the incision; during the operation, the wound was not sutured tightly, resulting in exudation and blood leakage; Wound infection. Wound healing includes two different forms: non physiological healing and physiological healing. Physiological healing refers to the replication of tissue cells according to their normal function, structure and morphology before injury, so as to achieve scar less healing and repair. Non physiological healing refers to the damaged tissue cells whose function, structure and morphology fail to return to normal, which belongs to scar repair ${ }^{[8]}$. The surface of a wound is a process of inflammatory reaction in wound healing; in this process, some immune cells play a very important role, such as clearing necrotic tissue, phagocytosis of invasive microorganisms, secretion of inflammatory mediators and so on $^{[9]}$. Wound healing includes three different stages: exudation, proliferation and scar formation ${ }^{[10]}$. Wound exudates after circumferential mixed hemorrhoids surgery contain many related factors, which can effectively activate the phagocytic system of the body, remove the necrotic tissue and bacteria in the wound, so as to achieve the inhibition of bacterial infection $^{[11]}$. The present study indicated that after the intervention, the quantitative scores of wound healing degree at different time points in the study group were lower than those in the control group $(\mathrm{p}<0.05$, respectively), The effective rate of the study group was significantly higher than that of the control group. The results showed that the combined use of titanoreine could significantly improve the effective rate, speed and quality of wound healing. The titanoreine is a kind of smooth and white suppository, which is torpedo shaped and has no special taste. The main active component of titanoreine is carrageenan, which is a kind of seaweed extract and can protect rectal mucosa. After application of titanoreine, a mucous membrane like structure will be formed and retained on the surface of rectal mucosa, which helps to prevent direct contact between rectal mucosa and rectal contents. In addition, titanoreine also contains semi synthetic solid glycerides, talc powder, zinc oxide and titanium dioxide, all of which can protect the mucosa and relieve mucosal congestion and convergence ${ }^{[12]}$. At the same time, semi synthetic solid glyceride can absorb water and swell in anus, and form a protective membrane together with other components of titanoreine, which can play a protective role and promote wound healing faster ${ }^{[13]}$. In this study, the average wound healing days in the study group were significantly shorter than those in the control group. The pathogenesis and causes of

TABLE 3: COMPARISON OF THE POSTOPERATIVE DEFECATION DIFFICULTY BETWEEN THE TWO GROUPS

\begin{tabular}{lcccc}
\hline Group & $\mathbf{n}$ & $\begin{array}{c}\text { First defecation } \\
\text { time (d) }\end{array}$ & $\begin{array}{c}\text { Quantitative score of constipation } \\
\text { symptoms 3 d after operation (points) }\end{array}$ & $\begin{array}{c}\text { Quantitative score of constipation } \\
\text { symptoms 7 d after operation (points) }\end{array}$ \\
\hline Control & 23 & $2.9 \pm 1.3$ & $11.1 \pm 0.9$ & $7.1 \pm 0.5$ \\
Study & 23 & $1.6 \pm 0.5$ & $8.2 \pm 0.7$ & $3.2 \pm 0.2$ \\
$\mathrm{t}$ & & 16.021 & 17.746 & 19.531 \\
$\mathrm{p}$ & & $<0.003$ & $<0.034$ & $<0.009$ \\
\hline
\end{tabular}


dysdefecation and constipation after circumferential mixed hemorrhoids operation include the following aspects: The patients were lack of activity because of fear of pain; Defecation can affect wound healing and induce pain, which makes patients unwilling to defecate and eventually become a vicious circle; The anesthetic drugs and damaged tissues during the operation will affect the intestinal motility; The lack of contents in the intestine cannot stimulate the intestinal wall, leading to the weakening of intestinal peristalsis and aggravation of constipation; Postoperative use of related drugs, such as analgesics and antibiotics, will have an impact on intestinal motility; Psychological factors; postoperative bad emotions, such as anxiety and fear, will affect normal defecation ${ }^{[14]}$. In this study, the first defecation time of the study group was significantly earlier than that of the control group $(p<0.05)$. The quantitative scores of constipation at different time points in the study group were significantly lower than those in the control group. The results showed that the combined use of titanoreine after operation can significantly improve the defecation difficulty of patients. The possible reason is that a layer of protective membranous structure formed after the use of titanoreine can fully isolate the intestinal contents, so as to avoid stimulating the injured mucosa, relieve the postoperative mucosal edema and pain, make the mucosa heal faster, relieve the obstruction feeling during defecation, and more easily discharge the stool ${ }^{[15]}$. At the same time, the semi synthetic solid glyceride and other related components in titanoreine can play a lubricating effect, which is more conducive to the discharge of stool. It can be concluded from the findings of this study that, the application of titanoreine after circular mixed hemorrhoids surgery can significantly shorten the time of wound healing, promote the wound healing faster and better, and reduce the risk of defecation difficulty.

\section{Acknowledgements:}

None

\section{Conflict of Interests:}

The authors declared no conflict of interest.

\section{REFERENCES}

1. Lihua Y, Xiao S. Effect of nursing mode combined with anal lifting exercise on visual analogue score of postoperative pain and rehabilitation process of circular mixed hemorrhoids. Chin Med Clin 2019;19(12):2139-40.

2. Longfang Q, Fang C, Xiaoqiang J. Clinical effect of Kangfuxin Liquid on wound healing of patients with mixed hemorrhoids after operation. Chin Med 2020;42(02):539-40.

3. Kestranek J. Hemorrhoid management in women: the role of tribenoside+lidocaine. Drugs Context 2019;8(3):1-7.

4. Guoguang G. Effect of Baiduobang combined with Kangfuxin liquid on wound surface and anal function of patients after low anal fistula operation. Colorectal Anal Surg 2018;24(01):60-3.

5. Yongliang Y, Feng Y, Pei Y. Effect of Kangfuxin Liquid Combined with Puji Zhichuang suppository on wound healing, anal function and quality of life after mixed hemorrhoids operation. Mod J Integr Chin West Med 2018;1(4):418-21.

6. Gomez-Mascaraque LG, Martinez-Sanz M, Fabra MJ, LopezRubio A. Development of gelatin-coated 1 -carrageenan hydrogel capsules by electric field-aided extrusion. Impact of phenolic compounds on their performance. Food Hydrocoll 2019;90(3):523-33.

7. Huo Bin. Effect of Jinxiao Zhuyu. Decoction on postoperative complications and wound healing of hemorrhoids. J Hubei Univ Tradit Chin Med 2020;109(02):83-6.

8. Jin Z, Zhengan Y. Effect of Cuyu Decoction on neovascularization and wound healing after circumferential mixed hemorrhoids operation. J Hunan Univ Tradit Chin Med 2019;39(02):253-6.

9. Moon SM, Lee SA, Hong JH, Kim JS, Kim DK, Kim CS. Oleamide suppresses inflammatory responses in LPSinduced RAW264. 7 murine macrophages and alleviates paw edema in a carrageenan-induced inflammatory rat model. Int Immunopharmacol 2018;56:179-85.

10. Liang H, Xiaolei LI, Cheng M. Effect of Longxuejie capsule combined with Jiuhua ointment treatment on wound after ring-like mixed hemorrhoid surgery. Int $\mathrm{J}$ Tradit Chin Med 2018;40(9):822-5.

11. Dayue P, Yangyi L, Xiangchun Z. Effect of rectal drip with self-made prescription on anal distension after operation of mixed hemorrhoids. Sichuan Tradit Chin Med 2019;2(8):16870 .

12. Terada A, Naruto S, Wachi K, Tanaka S, Iizuka Y, Misaka E. Synthesis and anti-inflammatory activity of [(cycloalkylmethyl) phenyl] acetic acids and related compounds. J Med Chem 1984;27(2):212-6.

13. Zeqin L, Huaqing S, Yunyun Z. Application effect of quot; washing, blowing and plugging and quot; three combined therapy in wound treatment after PPH. Chin J Basic Clin Pract Gen Surg 2019;8(6):691-5.

14. Bakry AM, Huang J, Zhai Y, Huang Q. Myofibrillar protein with $\kappa$-or $\lambda$-carrageenans as novel shell materials for microencapsulation of tuna oil through complex coacervation. Food Hydrocoll 2019;96:43-53.

15. Guohua X, Juan Q, Shaojun Z. Clinical observation of hemorrhoid point pricking therapy in the treatment of stage $\mathrm{I} \sim \mathrm{II}$ internal hemorrhoids .Chin $\mathrm{J}$ tradit Chin Med 2019;34(03):221-3. 